\title{
Frequency Spectra Fluctuations in the Radio Interferometry of Polarized Radiation
}

\author{
M.R.Olyak \\ Institute of Radio Astronomy of Ukrainian National Academy of \\ Sciences, 4 Krasnoznamennaya, Kharkov, Ukraine
}

\begin{abstract}
The presence of a longitudinal magnetic field is shown to lead to the appearance of an additional component of the frequency spectrum of the linearly polarized emission. This component is proportional to the spectrum of normal waves phase difference fluctuations. The fluctuations spectra baseline dependence of a linear polarized radiation is periodical near zero frequency, one is absent in the high frequency ranges and such dependence has different character in magnetoactive plasma with electron density irregularities and in plasma with a random magnetic field.
\end{abstract}

The effect of the non-relativistic electrons upon fluctuations of the $\mathrm{Q}$ and U Stokes parameters was studied by Simonetti, Cordes \& Spangler (1984) on the basis of the radiation transfer equation which, however, fails to allow for diffraction effects. At centimetre wavelengths, or in the case of optically thin sources, the method gives good results. At lower frequencies we obviously have to consider diffraction and, in particular, to include amplitude fluctuations in the analysis. And the Feynman integral technique using the solution to the parabolic equations govering the coherence matrix is more preferable for the analysis of mean - square and correlation function of the radiation at the output of plasma layer. Let consider the case of longitudinal propagation, when external magnetic field of strength $\vec{H}$ is directed along the $\mathrm{z}$-axis, $H_{0}$ is the mean component and $\delta H$ is the random component of the magnetic field. Let us assume that the following equations are valid: $\omega_{H} \ll \omega, \omega_{p} \ll \omega$, where $\omega_{p}$ and $\omega_{H}$ are the plasma and gyro frequency respectively. The wave of an arbitrary polarization can be decomposed in normal waves with right ( RCP ) and left ( LCP ) circular polarizations.

Let define the frequency spectra of the Stokes parameters fluctuations as $W_{i}(\vec{b}, f)=\int_{-\infty}^{\infty} d t \exp (2 \pi i f t) \Gamma_{i}\left(\vec{b}, \vec{v}_{\perp} t\right)$. Here $\vec{b}$ is the interferometer baseline, $\Gamma_{i}$ is the correlation function of either of components of the Stokes vector $\vec{S}=$ $\{I, Q, U, V\}, \vec{v}_{\perp}$ is the fluctuations transport speed in a plane perpendicular to the $\mathrm{z}$-axis.

The method of evaluation to $\Gamma_{i}$ was considered by Kukushkin \& Olyak (1994). They used the parabolic equation for Stokes parameters and the Feynman path-integral technique in the case when the transverse coherence scale $\left(R_{c}\right)$ is greater than the Fresnel-zone $\operatorname{size}\left(R_{F}=\sqrt{2 \pi z c / \omega}\right)$. Assume that the 
Markov process approximation and the hypothesis of the statistically uniform fluctuations of $\delta N$ and $\delta H$ are valid.

Let us put $\vec{b}=0$ and assume the source to be point-like over the angular coordinates and the initial Stokes vector to take value $\vec{S}_{0}=\left\{I_{0}, Q_{0}, U_{0}, V_{0}\right\}$.The fluctuations in the electron density and the magnetic field are statistically independent. Take a Kolmogorov model for the spectra of $\delta N$ and $\delta H$ with a power index of $\nu$, inner turbulent scale $l_{0}$, structure constants of relative fluctuations of $\delta N / N$ and $\delta H / H_{0}$ are respectively $C_{N}^{2}$ and $C_{H}^{2}$. Suppose the following inequalities hold $c z / \omega \gg l_{0}^{2}, z c \omega_{H} \omega_{p}^{2} / \omega^{4} \ll l_{0}^{2}$. Last equation implies that the difference in Fresnel-zone sizes for RCP and LCP waves is less than the inner scale of the irregularities, and hence the ray trajectories of the RCP and LCP waves actually coincide. Put $\Omega=f / f_{0}$, where $f_{0}=\sqrt{v_{\perp}^{2} \omega / 4 \pi^{2} z c}$. In the case of small fluctuations of the phase difference, with the mean-square phase difference of RCP and LCP waves $<\left(\delta S^{+}-\delta S^{-}\right)^{2}>\ll 1$, the equation for linear polarized fluctuating radiation spectra $W_{l}=W_{Q}+W_{U}$ is given by

$$
\begin{aligned}
W_{l}(\Omega)= & \left\{\left(C_{N}^{2}+4\left(\omega_{H} / \omega\right)^{4} C_{H}^{2}\right) G_{\chi}(\Omega)+\right. \\
& \left.+\left(\omega_{H} / \omega\right)^{2}\left(C_{N}^{2}+C_{H}^{2}\right) G_{S}(\Omega)\right\}\left(Q_{0}^{2}+U_{0}^{2}\right)
\end{aligned}
$$

where $G_{\chi}(\Omega)$ and $G_{S}(\Omega)$ are similar to the amplitude and phase fluctuations spectra of Tatarskii (1971).

Let baseline $\vec{b} \| \vec{v}$. For frequency spectrum of linearly polarized fraction we can obtain

$$
\begin{aligned}
W_{l}(\vec{b}, \Omega)= & W_{l}(\Omega)+\left\{C_{N}^{2}-\left(\omega_{H} / \omega\right)^{2} C_{H}^{2}\right\}\left\{G_{S}(\Omega)-\right. \\
& \left.-G_{\chi}(\Omega)\right\} \sin ^{2}\left(\pi b f_{0} \Omega / v_{\perp}\right)\left(Q_{0}^{2}+U_{0}^{2}\right)
\end{aligned}
$$

when $W_{l}(\Omega)$ is determined by $(1)$.

As can be seen from (2), the baseline dependence of the fluctuations spectrum of the linear polarized fraction has periodical character near zero frequency when $G_{\chi}(\Omega) \not \equiv G_{S}(\Omega)$. In the rest frequency ranges the spectrum dependence of an interferometer baseline is absent owing to $G_{\chi}(\Omega)=G_{S}(\Omega)$ for $\Omega \gg 1$ (Tatarskii, 1971). Also we can see from (1) and (2) that the frequency dependences of $W_{l}(\Omega)$ and $W_{l}(\vec{b}, \Omega)$ have different character in plasma with electron density irregularities and in plasma with a random magnetic field . In the last case the presence of a longitudinal component of a random magnetic field is shown to lead to the appearance of an additional component of $W_{l}(\Omega)$ and a negative sign of the last term in (2). This component is proportional to the phase difference spectrum of the fluctuations of the ordinary and extraordinary waves.

This research was partially supported by a grant INTAS-CNES 97-1450.

\section{References}

Kukushkin, A.V., \& Olyak, M.R. 1994, Waves in Random Media, 4, 59

Simonetti, J.H., Cordes, J.M. \& Spangler, S.R. 1984, ApJ, 284, 126

Tatarskii, V.I. 1971, The Effects of the Turbulent Atmosphere on Wave Propagation (U.S.Department of Commerce. Washington.D.C.) 